\title{
Plucked piezoelectric bimorphs for energy harvesting applications
}

\author{
Michele Pozzi* and Meiling Zhu \\ Department of Materials, School of Applied Sciences \\ Cranfield University, Bedfordshire, MK43 0AL, UK
}

\begin{abstract}
The modern drive towards mobility and wireless devices is motivating intense research in energy harvesting (EH) technologies. In an effort to reduce the battery burden of people, we are investigating a novel piezoelectric wearable energy harvester. As piezoelectric $\mathrm{EH}$ is significantly more effective at high frequencies, in opposition to the characteristically low-frequency human activities, we propose the use of an up-conversion strategy analogous to the pizzicato musical technique. In order to guide the design of such harvester, we have modelled with Finite Elements (FE) the response and power generation of a piezoelectric bimorph while it is "plucked", i.e. deflected, then released and permitted to vibrate freely. An experimental rig has been devised and set up to reproduce the action of the bimorph in the harvester. Measurements of the voltage output and the energy dissipated across a series resistor are reported and compared with the FE predictions. As the novel harvester will feature a number of bimorphs, each plucked tens of times per step, we predict a total power output of several $\mathrm{mW}$, with imperceptible effect on the wearer's gait.
\end{abstract}

Keywords: Plucked piezoelectric bimorphs, frequency up-conversion, energy harvesting, human gait, knee harvester, wearable energy harvesting, pizzicato, portable power

\section{INTRODUCTION}

As the power consumption of electronic devices decreases, following greater integration and miniaturization, the prospect of powering such devices relying exclusively on harvested power becomes more and more feasible. Initial research focused on environmental energy scavenging, for application in wireless sensor nodes dedicated to monitoring environments and structures. More recently, increasing research efforts have been directed towards human-based EH, aiming at powering consumer devices. An excellent example of energy harvesting from the mechanical dissipative work taking place during normal gait was presented in $2008^{1}$. The authors developed an electromagnetic device capable of producing $4.8 \pm 0.8 \mathrm{~W}$ in the generative-braking mode during human walking. In this paper, we study a lighter and mechanically less complex device inspired by similar principles but based on piezoelectric bimorphs, which are plucked while the wearer is simply walking.

Human activities typically occur at very low frequencies, up to a few hertz. As is well known, piezoelectric bimorphs operating for sensing or energy generation are most effective at much higher frequencies. Due to the large stiffness of piezoelectric ceramics, harvesters with power outputs in the order of milliwatts, and having dimensions and mass acceptable for human integration, have resonance frequencies of hundreds of hertz. This frequency mismatch represent an obstacle to the development of an efficient piezoelectric energy-harvester for human integration.

The solution adopted in this paper is the implementation of a frequency up-conversion strategy similar to the pizzicato technique used in music. Several concept designs for energy-harvesters based on mechanical or magnetic up-conversion were presented by Rastegar and Murray ${ }^{2}$. In all designs, the fundamental principle is the transfer of energy from a "primary system", directly coupled to a low frequency input, to a "secondary vibrating system" with higher resonant frequency. Sari et al. ${ }^{3}$ have applied this principle of frequency up-conversion to the microscale and prototyped an electromagnetic generator where the low-frequency vibration of a suspended magnet excites the high frequency vibration

* e-mail: m.pozzi@cranfield.ac.uk (corresponding author) 
of a number of coil-carrying cantilevers placed around it. An interesting piezoelectric harvester not based on seismic oscillation but rather on the direct deformation of cantilevers was presented in 2005 by Priya ${ }^{4}$. Here the piezoelectric bimorphs oscillate between stops which are mounted on a shaft set into motion by an air flow, as in a conventional windmill.

In the present work, we propose the use of a plucking-based frequency up-conversion strategy for energy harvesting on humans, with special focus on converting the low-frequency rotation of the knee joint into high-frequency vibration of a number of piezoelectric bimorphs. We developed a Finite Element Model to study the response and energy production of a bimorph which is plucked, i.e. slowly deformed and then released to permit free vibrations. It should be mentioned that most of the literature on piezoelectric energy harvesting is still devoted to vibrational energy scavenging. As a result, it is possible to find many models, both analytical ${ }^{5}$ and based on finite elements ${ }^{6}$, where the cantilevers, most typically with an added mass on the tip, are subject to seismic excitation, usually of harmonic character. To the best of the authors' knowledge, the input of mechanical energy into a piezoelectric EH device by sporadic mechanical plucking has never been modelled before. Yet, such analyses are necessary to identify the many design parameters of a mechanical upconversion harvester, such as piezoelectric material, frequency of plucking actions, dimensions of the piezoelectric bimorphs, number and spacing of the plectra that deflect the bimorphs and so on.

\section{FREQUENCY UP-CONVERSION BY MECHANICAL PLUCKING}

The plucking strategy here proposed for frequency up-conversion is very similar in principle to the pizzicato technique used in chorded musical instruments like the harpsichord or the guitar. As Figure 1 shows, mechanical plucking is constituted of a sequence of three main phases. In the approach phase, the distance between bimorph and plectrum is reduced until contact is made. Immediately follows the loading phase, during which both elastic elements are deflected, according to their mechanical compliance and energy is stored elastically in the structures. As the deflection progresses, the area of overlap between the two elements is reduced. A limiting deflection is reached when contact is lost and each element is free to spring back to its original shape; this is the release point. From this instant on, the bimorph vibrates at its resonance frequency around its rest position as a cantilever beam. As the bimorph vibrates, part the mechanical energy input during deflection is converted by the direct piezoelectric effect into electrical energy and transferred to the external circuit; the rest of the mechanical energy is dissipated through various mechanisms, like air damping, dielectric losses and material internal damping. The outcome of plucking is frequency up-conversion, as by one single slow movement of the plectrum a large number of vibrations are produced at high frequency.
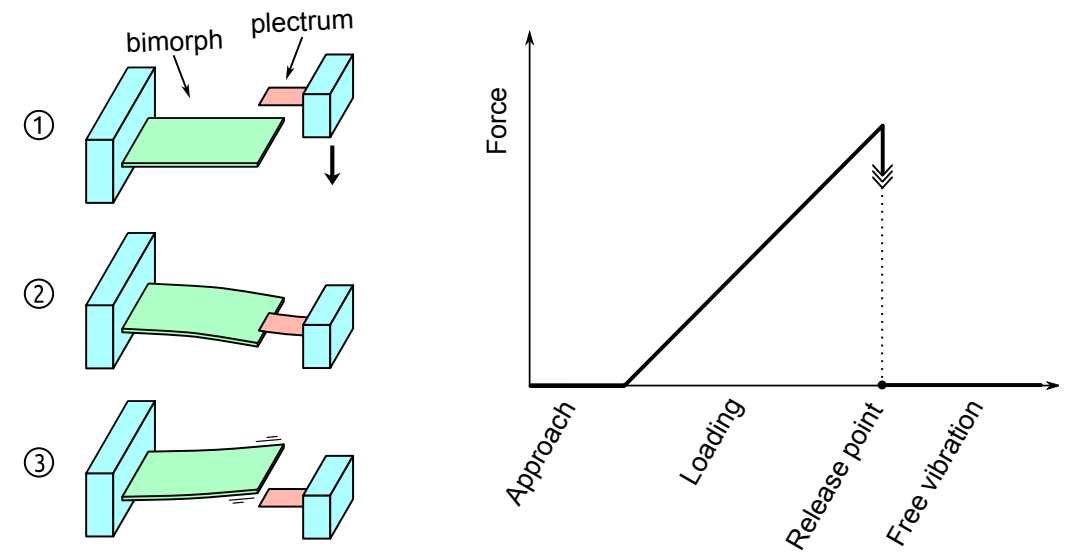

Figure 1. Illustration of a plucking event: 1) approach; 2) loading step with deflection of bimorph and plectrum; 3) release step followed by vibration of the bimorph at its resonance frequency. The plot depicts the evolution of the external force applied at the tip of the bimorph as the plucking action progresses. 
A knee-joint harvester that ensures the repetition of the plucking actions to achieve sustained power production is sketched in Figure 2. During normal gait, for example while walking, the knee alternatively bends and extends once per second, covering a rotation angle of approximately 70 degrees. With reference to the figure, the outer ring, carrying the plectra, is fixed to the thigh, whilst the internal hub, carrying the bimorphs, rotates with the knee as it is fixed to the lower leg. As the person walks and the knee alternatively extends and bends, each of the multiple plectra on the outer ring plucks the bimorphs according to the sequence outlined in Figure 1. The energy harvester is therefore capable of converting the slow motion of walking into high-frequency vibrations of several piezoelectric bimorphs. It is expected that the design have the potential to produce a sustained power in the order of milliwatts during walking.

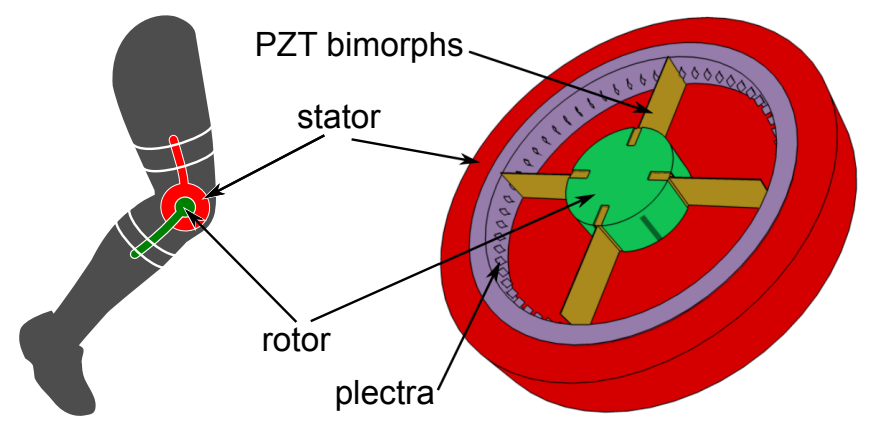

Figure 2: Knee-joint piezoelectric harvester. It is worn on the external side of the knee and fixed by braces. Inside, a hub carries a number of bimorphs which are plucked by the ring-mounted plectra as the joint rotates during normal walking.

\section{MODELLING APPROACH AND EXPERIMENTAL METHODS}

\subsection{Finite Element Modelling}

Finite Element (FE) modelling was performed using the commercial package ANSYS 11.0 (ANSYS Inc. Canonsburg, PA, USA). The analysis is based on SOLID5 piezoelectric elements, SOLID45 structural elements and uses CIRCU94 elements for the resistor placed across the electrodes of the bimorph. The boundary conditions include displacement constraints on the top and bottom surfaces in the clamped portion of the beam. The device modelled is a bimorph having a brass substrate sandwiched between two sheets of PZT; the device is poled for series operation and the electrodes fully cover the top and bottom surfaces (details of geometry and material properties in Table 1). The model includes viscous damping, whose coefficients were deduced from the tabulated mechanical quality factors $\mathrm{Q}_{\mathrm{m}}$.

A transient analysis was performed to study the deflection and subsequent free vibration of the bimorph. The analysis was divided into two distinct steps. The first is a loading step, during which the bimorph was deflected at constant speed to a predefined tip displacement. In the FE model, this was achieved with a ramped load solution step. This approximates the deflection produced on the bimorph by a plectrum moving at constant speed, as seen in Figure 1. The following step is the release phase and was modelled in a second transient analysis, starting with a stepped removal of the tip constraints of a statically deflected beam.

To assess the energy production of the bimorph, the instantaneous power $P\left(t_{i}\right)$ at substep $i$ was calculated from the voltage across the resistor at the same substep:

$$
P\left(t_{i}\right)=\frac{V^{2}\left(t_{i}\right)}{R}
$$


The instantaneous power is then used to calculate the cumulative energy produced up to substep $n$ :

$$
E\left(t_{n}\right)=\sum_{i=0}^{n} P\left(t_{i}\right) \Delta t_{i}
$$

where $\Delta t_{i}$ is the duration of each substep. Naturally, $E\left(t_{n}\right)$ will be a monotonic function of time.

Table 1. Geometrical parameters of the model and materials properties.

\begin{tabular}{|c|c|c|c|}
\hline \multicolumn{2}{|c|}{ Geometry } & \multicolumn{2}{|c|}{ Material properties (PZT-5H) } \\
\hline Total length & $30 \mathrm{~mm}$ & Density & $7800 \mathrm{~kg} / \mathrm{m}^{3}$ \\
\hline Free length & $25 \mathrm{~mm}$ & $\mathrm{c}^{\mathrm{E}}{ }_{11}$ & $62 \mathrm{GPa}$ \\
\hline Width & $10 \mathrm{~mm}$ & $\mathrm{c}^{\mathrm{E}}{ }_{33}$ & $50 \mathrm{GPa}$ \\
\hline Total thickness & $400 \mu \mathrm{m}$ & $d_{31}$ & $-32010^{-12} \mathrm{~m} / \mathrm{V}$ \\
\hline \multirow[t]{4}{*}{ PZT thickness } & $150 \mu \mathrm{m}$ & $d_{33}$ & $65010^{-12} \mathrm{~m} / \mathrm{V}$ \\
\hline & & $\varepsilon^{\mathrm{T}}{ }_{11}$ & 3600 \\
\hline & & $\varepsilon_{33}^{\mathrm{T}}$ & 3800 \\
\hline & & $\mathrm{Q}_{\mathrm{m}}$ & 32 \\
\hline
\end{tabular}

\subsection{Experimental details}

The closest match to the bimorph specified in Table 1 available commercially was found in the piezoelectric bimorphs type T215-H4-303X produced by Piezo Systems Co.; their dimensions are $31.8 \times 12.7 \times 0.38 \mathrm{~mm}^{3}$, they are made of PZT$5 \mathrm{H}$ and they are poled for series operation. As for mechanical construction, a $130 \mu \mathrm{m}$-thick metal shim is sandwiched between two layers of PZT, each of thickness $125 \mu \mathrm{m}$.

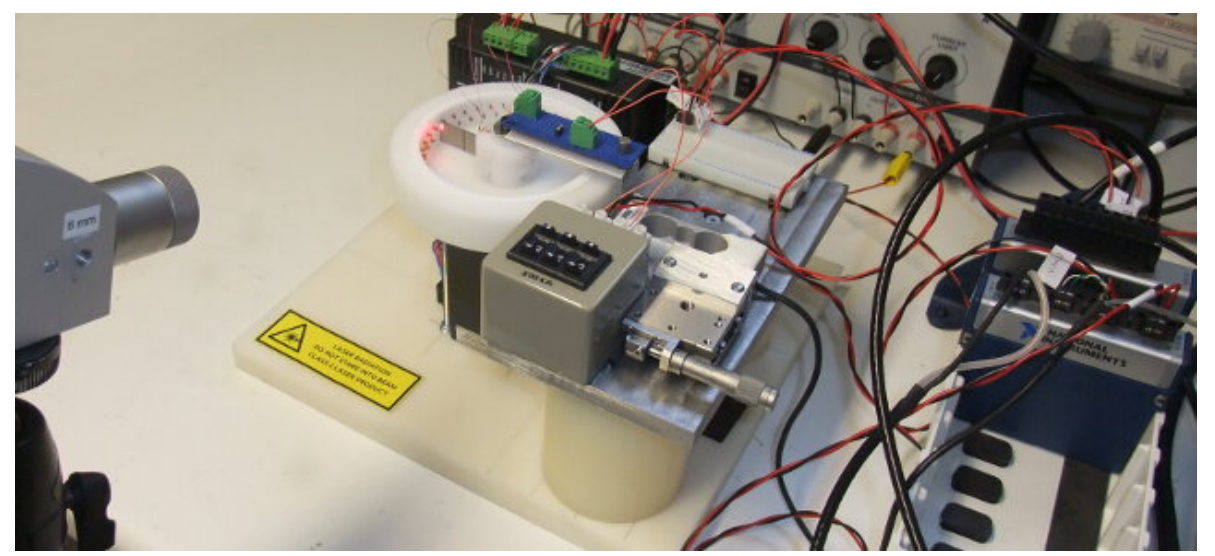

Figure 3: Experimental rig developed to reproduce the motion of the knee. The outer ring is fixed to the shaft of the stepper motor; the central hub is held by a metallic arm connected to a load cell. The grey box in the foreground contains the variable resistor. On the left, the objective of the LDV is visible. In the photograph, only one bimorph is mounted, although there are 4 mounting slots in the hub.

An experimental rig was developed, which permits to reproduce the rotation of the knee-joint (Figure 3). A stepper motor is connected to the outer ring, carrying the plectra. The inner hub is kept still by a support arm. This mechanical configuration isolates the bimorphs from the vibrations produced by the stepper motor and permits the measurement of the torque experienced by the hub (useful to estimate the degree of comfort of the harvester). The steps for the motor are produced by a stepper driver, in turn controlled by a computer, so that any speed/rotation profile compatible with mechanics and electronics limits can be reproduced. 
The root of the bimorph is clamped within copper-clad plates leaving a free length of $26 \mathrm{~mm}$. The plectra have the shape of a $3 \times 2 \mathrm{~mm}^{2}(l \times w)$ rectangle and are cut out of a $125 \mu$ m-thick Kapton ${ }^{\circledR}$ polyimide film. The terminals of the bimorph were connected to a selection of electrical loads; the voltage across such load was digitally sampled and transferred to a computer. A Laser Doppler Vibrometer (Polytec CLV-2534) targeted onto the tip of the bimorph was used to measure the velocity of vibrations.

\section{RESULTS AND DISCUSSION}

\subsection{Simulated transient analysis: loading step}

In real operation, the proposed harvester would experience variable rotational speeds, as the angular velocity of the kneejoint changes during the gait cycle. Geometrical factors, in particular the radius of the plectra-carrying ring, determines how this angular rotation is translated into relative velocity between plectra and bimorphs. Therefore, it is important to understand how the speed of deflection influences the energy produced. With this aim, the transient analyses for the loading step were conducted with different deflection speeds.

What emerges from these analyses is that, having fixed the load resistor, the energy produced decrease as the deflection speed decreases (Figure 4). In fact, if we assume that the total charge Q produced by deflecting the piezoelectric material depends only on the final strain, so in particular we disregard the higher modes of vibration which are introduced by the ramped deflection, we have:

$$
I=\frac{Q}{\Delta t} \text { and so: } P=R I^{2}=\frac{R Q^{2}}{\Delta t^{2}} \text { or also: } E=\frac{R Q^{2}}{\Delta t}
$$

This relation between the energy $\mathrm{E}$ and the deflection time $\Delta t$, was asymptotically observed by the modelling results for slow deflections, as the higher modes become less important (Figure 4).

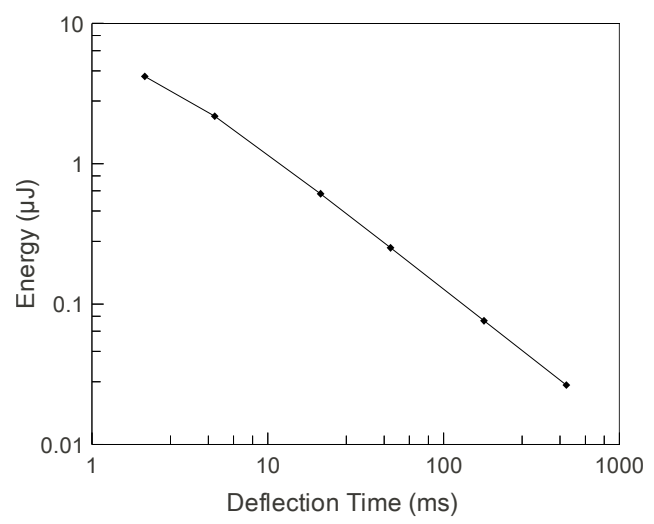

Figure 4. Predicted effect of the speed of deflection on the total energy produced in the loading step across a $20 \mathrm{k} \Omega$ resistor and with maximum deflection of $0.5 \mathrm{~mm}$. The curve asymptotically approaches a straight line as the deflection time increases, in agreement with Equation 3

\subsection{Simulated transient analysis: release step}

Perhaps the most interesting outcome of the FE analyses, and certainly the most useful results in view of the intended application, pertain to the behaviour of the cantilever upon release. With a full transient analysis, we analysed the vibrations of the piezoelectric device and looked at the power output at every substep, according to Equation 1, from this we calculated the time evolution of the energy produced. 
After being deflected very slowly by $0.5 \mathrm{~mm}$, the cantilever is released at time $1 \mathrm{~s}$ and its dynamics is followed for several milliseconds. The deflection was set to occur over $1 \mathrm{~s}$ to ensure that velocities are zero at the time of release (the time axis starts at $1 \mathrm{~s}$ in the graphs as a reminder of this). The many curves in Figure 5 (cumulative energy versus time, calculated according to Equation 2) refer to different electrical loadings, from $1 \Omega$ to $500 \mathrm{k} \Omega$, as specified on the plot. The bimorph can produce up to $59 \mu \mathrm{J}$ across $20 \mathrm{k} \Omega$ (Figure 5).

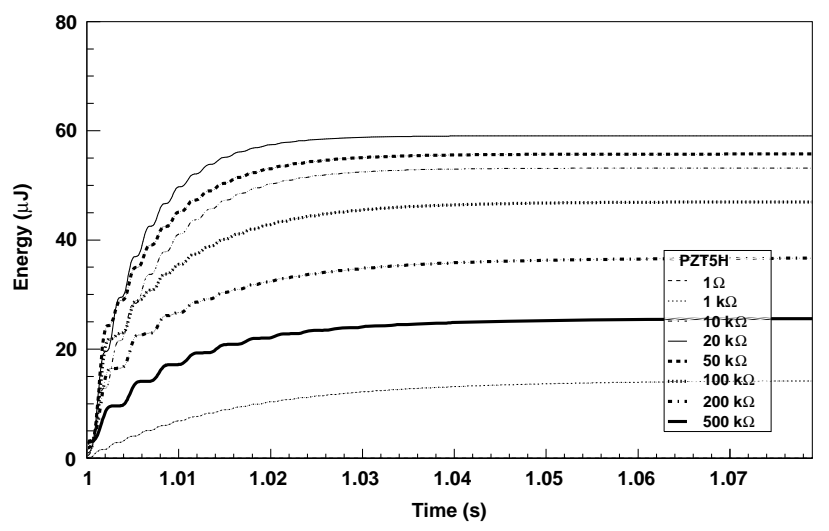

Figure 5. Predicted cumulative energy produced by the bimorph upon release after a deformation of $0.5 \mathrm{~mm}$ lasting $1 \mathrm{~s}$. The slow deformation ensures that the bimorph is not vibrating at the time of release.

Although the largest energy is obviously produced from each plucking event after an "infinite" time, the best strategy to maximize the overall power production of a practical harvester is to repeatedly pluck the bimorph, while it is still vibrating. The plots in Figure 5 can be used to optimise the repetition time of plucking actions. However, as human activities are not perfectly repeatable, any human-integrated device is likely to operate over a wide range of conditions; this means that it is not possible to design a harvester working constantly at the optimal plucking frequency.

\subsection{Experimental plucking events}

Experimental data were collected using the setup described earlier with the aim of validating the model. The device voltage output and the velocity of the tip following a plucking event are well predicted by the model (Figure 6). For the comparison, the geometrical parameters of model presented in the previous sections were adapted to the real device. We have also determined the mechanical quality factor $Q_{m}$ by fitting a damped second order response to the measurements in open circuit conditions and then extracting $Q_{m}$ from the exponential decay. The value calculated in this way differed slightly from that tabulated on the supplier's datasheet for the material $\left(Q_{m}=35\right.$ and $Q_{m}=32$, respectively). As for the initial conditions, it was not possible to reliably measure the deflection of the tip before release. Thus we have set the tip displacement in the FE model so that the first predicted velocity peak had equal value to the corresponding peak in the data from the LDV (see reference point marked on Figure 6). A deflection of $88 \mu \mathrm{m}$ satisfies this requirement. However, time integration of the velocity showed that the maximum deflection experienced by the bimorph before release was about $120 \mu \mathrm{m}$, suggesting that for the first $\sim 30 \mu \mathrm{m}$ the bimorph is still in partial contact with the plectrum and is not released neatly and quickly. This issue is visible in the neighbourhood of the release point ( $t=0 s$ in Figure 6), where we can see that the velocity of the bimorph initially increase rather slowly and with considerable noise, due to the rubbing between plectrum and bimorph. The voltage curves show a measurable output before $t=0 \mathrm{~s}$, and it is therefore not surprising that the the model predicts less energy than is measured: in those initial $\sim 30 \mu \mathrm{m}$ some energy is produced because the bimorph is moving, albeit not freely.

These tests show an energy generation of $2 \mu \mathrm{J}$; other tests, with larger deflections of the bimorph, have produced approximately $40 \mu \mathrm{J}$. As regards the frequency of oscillations, the agreement between model and experiment is very good with $294 \mathrm{~Hz}$ (model) vs. $291 \mathrm{~Hz}$ (experiment). 


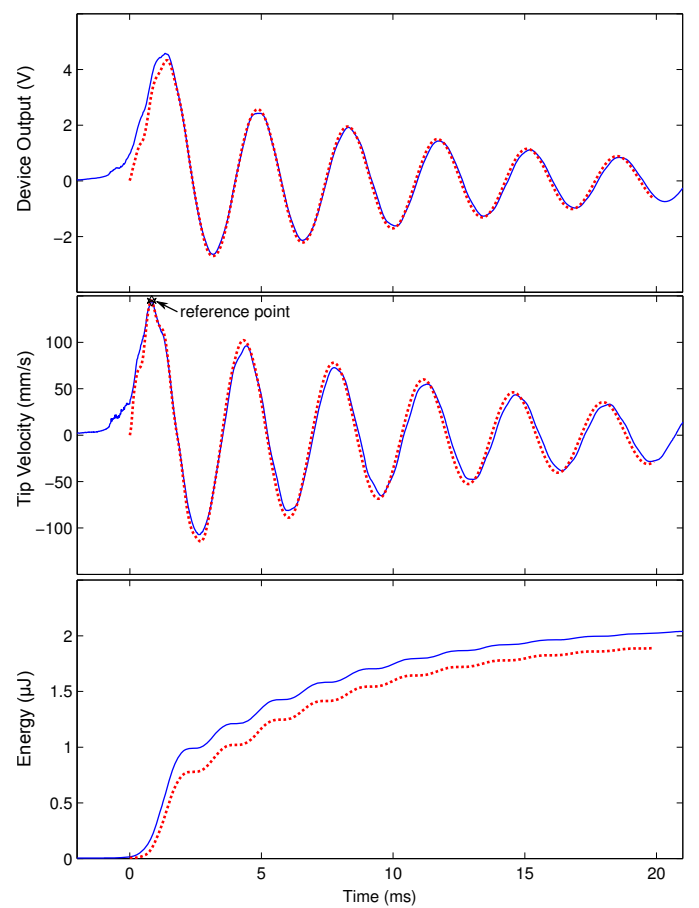

Figure 6. Comparison between experimental results (solid blue line) and model (dashed red line). In the plots: voltage measured across a $25 \mathrm{k} \Omega$ resistor, velocity of the tip and electrical energy dissipated by the resistor during a single plucking event. The symbol on the velocity plot indicates the point at which the model output velocity was matched to the experimental measurement.

\section{CONCLUSIONS}

Piezoelectric energy harvesters often have to battle with contrasting frequency requirements: the available vibrations are low-frequency, but the harvester's active element performs best at high frequencies. For this reason, we adopted mechanical plucking as the frequency up-conversion strategy for human-based EH. Bimorph plucking means that a piezoelectric bimorph is deflected by mechanical contact with a plectrum so that, when rapidly released, it may vibrate around its rest position at its own resonant frequency. During these vibrations, an important portion of the elastic energy stored is converted into electrical energy.

In this paper we have presented a finite element model to simulate the response of a plucked bimorph, predicting voltage and energy dissipated across a resistor placed in series to the device. Although frequency up-conversion strategies have previous reports in the literature, as outlined in the introduction, it is the first time that the plucking mode of operation is systematically studied and modelled, with a view to human based energy harvesting. The results of this model give needed insight for the design of energy-harvesters based on plucking as the frequency up-conversion strategy.

We have reported experimental measurements to validate the model. A slight mismatch between model and experiment velocity data at the time of release has allowed us to identify the action of releasing the bimorph as a potentially major source of energy loss. More specifically, if the release is not sufficiently clean, as happens when the contact between plectrum and bimorph is lost over a significant distance of relative motion, the initial deflection of the bimorph cannot be fully exploited for energy generation. 


\section{ACKNOWLEDGEMENTS}

This research is sponsored by the Engineering and Physical Sciences Research Council (EPSRC) via grant No. $\mathrm{EP} / \mathrm{H} 020764 / 1$.

\section{REFERENCES}

[1] Donelan, J.M., Li, Q., Naing, V., Hoffer, J.A., Weber, D.J., and Kuo, A.D., "Biomechanical Energy Harvesting: Generating Electricity During Walking with Minimal User Effort,” Science 319(5864), 807-810 (2008).

[2] Rastegar, J., and Murray, R., "Novel two-stage piezoelectric-based electrical energy generators for low and variable speed rotary machinery," in Proceedings of SPIE, 72880B-72880B-8 (2009).

[3] Sari, I., Balkan, T., and Kulah, H., "An Electromagnetic Micro Power Generator for Low-Frequency Environmental Vibrations Based on the Frequency Upconversion Technique," Microelectromechanical Systems, Journal of 19(1), 14-27 (2010).

[4] Priya, S., "Modeling of electric energy harvesting using piezoelectric windmill," Applied Physics Letters 87(18), 184101 (2005).

[5] Erturk, A., and Inman, D.J., "A Distributed Parameter Electromechanical Model for Cantilevered Piezoelectric Energy Harvesters," Journal of Vibration and Acoustics 130(4), 041002-15 (2008).

[6] Zhu, M., Worthington, E., and Njuguna, J., "Analyses of power output of piezoelectric energy-harvesting devices directly connected to a load resistor using a coupled piezoelectric-circuit finite element method," Ultrasonics, Ferroelectrics and Frequency Control, IEEE Transactions on 56(7), 1309-1317 (2009). 